multidisciplinary team (MDT) supported listing in $70 / 184$ (38\%). 105/184 (57\%) were not listed and 9/184 (5\%) did not complete the assessment. Of the $57 \%$ not listed, $33 \%$ had an improving clinical trajectory or alternative treatment option e.g. TIPSS. $24 \%$ were too high risk due to comorbidity and $17 \%$ were thought to have too high a risk of dependency relapse. However, 19\% required further optimisation for frailty or malnutrition and had their assessment conclusions deferred.

Conclusion Our data show that one fifth of patients assessed for $\operatorname{ArLD}$ require optimisation which prevents their listing. Such candidates are usually frail and/or malnourished and represent late stage disease. These may be surrogate markers of late presentation or late referrals e.g. in the case of $>5$ paracenteses. New BSG guidelines in 2020 recommend early referral (at three months if no improvement, or earlier if urgent late presentations) for patients with decompensated ArLD. This enables alcohol and allied health specialists additional time to work with patients in order to achieve favourable outcomes. This should provide reassurance and empower referring clinicians, especially those that do not have access to specialist alcohol services in their units.

\section{PWE-26 NORTHERN IRELAND EXPERIENCE OF KAFFES STENTS USAGE FOR BILIARY ANASTAMOTIC STRICTURES POST LIVER TRANSPLANT}

${ }^{1}$ Rebecca O' Kane, ${ }^{2}$ Rebecca O' Kane*, ${ }^{3}$ Neil Mc Dougall. 'Gastroenterology Registrar, Belfast, UK; ${ }^{2}$ Gastroenterology Registrar, Belfast, UK; ${ }^{3}$ Consultant Hepatologist, Belfast, United Kindgom

\subsection{6/gutjnl-2021-BSG.216}

Introduction Biliary anastomotic strictures are isolated, shortlength strictures that occur in $10-16 \%$ of patients post orthotopic liver transplantation (OLT). The strictures are most common in the first year post OLT but can occur at any time. Management has historically been with repeated dilations and plastic stenting and if this fails to resolve the stricture, hepaticojejunostomy is considered.

Kaffes stents (Taewoong Medical) are a novel type of fully covered self expanding metallic stent with a design that helps reduce the risk of stent migration. Previous randomised trials have illustrated their success with resolving anastomotic strictures when compared to plastic stenting.

Methods The Regional Liver Unit began using Kaffes stents to manage anastomotic strictures in 2018.

A Kaffes stent was used for all post OLT biliary anastomotic strictures that were causing derangement of LFTs provided that there was sufficient space to place the upper end of the stent below the hilum.

The stent was left in situ for 8-12 weeks and second stent inserted at repeat ERCP if stricture not fully resolved. Biliary reconstruction discussed with OLT surgeon if stricture not resolved after 2 stents.

A retrospective analysis was carried out of all OLT recipients who had a Kaffes stent inserted. Patient list retrieved from electronic liver transplant database. Data obtained from ERCP reports and recipient electronic care records.

Results From May 2018 to Aug 2020, nineteen OLT patients had a Kaffes stent inserted.

The median time to stricture diagnosis was 4.5 months (shortest: 0.5 months, longest 147 months). 8 developed a stricture within 3 months, 5 with 12 months and 6 more than 12 months after transplant.

11 had no previous intervention for their stricture, with remainder having previous plastic stent insertion (prior to Kaffes stent becoming available)

Stents were inserted for a median of 12.5 weeks. Two sizes of stent were used: $40 \mathrm{~mm}$ length and either $8 \mathrm{~mm}$ or $10 \mathrm{~mm}$ width.

$15(79 \%)$ patients achieved resolution of the stricture - 10 requiring just one stent, 4 requiring a second stent and 1 requiring a third stent.

4 patients failed to resolve with Kaffes stent - 1 has been relisted for further liver transplant and is undergoing dilatation and straight stents 3 monthly, 1 undergoes intermittent dilatations/plastic stents (not fit for surgery), 1 underwent a hepaticojejunostomy and 1 has been listed for biliary reconstruction surgery.

Conclusions The Kaffes stent is effective in resolving biliary anastomotic strictures post-OLT for over $75 \%$ of patients, with the majority just requiring a single Kaffes stent. The reduced requirement for repeat ERCPs should more than offset the cost of a Kaffes stent compared to a plastic stent.

\section{PWE-27 PROFILE OF THE USE OF STATINS IN RELATION TO LIVER FUNCTION TESTS IN CARDIOLOGY PATIENTS}

Sonia Moteea*, Jessica Morrison, Carol Firth, Sarah Tinker, Sulleman Moreea. Bradford Teaching Hospital, Leeds, UK

\subsection{6/gutjnl-2021-BSG.217}

Background According to NICE prior to starting statin therapy a patient should have liver function tests (LFTs) specifically alanine aminotransferase (ALT) performed ${ }^{1}$. LFTs should be repeated at three and twelve months post starting statin therapy ${ }^{1}$. Statins are frequently associated with elevated LFTs, especially during first weeks of treatment. However, they are rarely directly associated with causation of severe liver disease $^{2}$. Emerging evidence suggests LFT monitoring for statin therapy is overdone and costly ${ }^{3}$.

Aims To review the effect of statin therapy on ALT results in Cardiology patients.

Method For the period Jan 2015-Dec 2017 (36 months), we extracted data for all patients admitted under cardiology from our electronic databases: patient demographics; diagnosis on discharge, medication on discharge; LFTs before and after starting statins.

Result During the study period, 4871 patients were admitted with a diagnosis of acute coronary syndrome (ACS). 729 (15\%) patients (456 M, mean age 62; $273 \mathrm{~F}$, mean age 66) died during their admission. Of the remaining 4142 patients (2677 M, mean age 64; $1465 \mathrm{~F}$, mean age 68), 1854 (45\%) (1312 M, mean age 60; F 542, mean age 65) where commenced on statin therapy. 654(35\%) patients (188 F, $457 \mathrm{M})$ had LFTs measured on admission. Of these 499 (76\%) (151 F, $348 \mathrm{M})$ had a normal ALT at baseline - 365 (73\%) had normal ALT on repeat, 95 (19\%) had no repeat test and 39 (8\%) had abnormal ALT on repeat. Of the 145 (24\%) (37 F, $108 \mathrm{M})$ with raised ALT at baseline, 18 (12\%) had no repeat ALT, 86 (59\%) had normal ALT on repeat testing and 42 (29\%) had persistently raised ALT. Of the latter $22(15 \%)$ had an ALT worse than at baseline. 13/654 (2\%) had ALT > 450 (X10 ULN) during repeat testing - related to alternative 